\section{Organic-Based Photovoltaics: Toward Low-Cost Power Generation}

\author{
Sean E. Shaheen, David S. Ginley, and \\ Ghassan E. Jabbour, Guest Editors
}

\begin{abstract}
Harvesting energy directly from sunlight using photovoltaic technology is a way to address growing global energy needs with a renewable resource while minimizing detrimental effects on the environment by reducing atmospheric emissions. This issue of MRS Bulletin on "Organic-Based Photovoltaics" looks at a new generation of solar cells that have the potential to be produced inexpensively. Recent advances in solar power conversion efficiencies have propelled organic-based photovoltaics out of the realm of strictly fundamental research at the university level and into the industrial laboratory setting. Fabricated from organic materials-polymers and molecules-these devices are potentially easier to manufacture than current technologies based on silicon or other materials. In this introductory article, we describe the motivation for pursuing research in this field and provide an overview of the various technical approaches that have been developed to date. We conclude by discussing the challenges that need to be overcome in order for organic photovoltaics to realize their potential as an economically viable path to harvesting energy from sunlight.
\end{abstract}

Keywords: electron acceptors, energy production, excitons, metal oxide semiconductors, nanostructures, organic semiconductors, photovoltaics, polymers, power generation, quantum dots, solar cells.

\section{Introduction}

Harvesting energy directly from sunlight using photovoltaic (PV) technology* is being increasingly recognized as an essential component of future global energy production. The finite supply of fossil fuel sources and the detrimental long-term effects of $\mathrm{CO}_{2}$ and other emissions into our atmosphere underscore the urgency of developing renewable energy resources. PV technology is being increasingly recognized as part of the solution to the growing energy challenge and an essential component of future global energy production. Pro-

\footnotetext{
*An introduction to photovoltaic technology can be found at Web site www.eere.energy.gov/ solar/pv_basics.html.
}

vided that photovoltaics can be made truly economically competitive with fossil fuels, large-scale manufacturing of these devices offers a pathway to a sustainable energy source that could supply a significant fraction of our energy needs. This comes with only minimal impact on the environment associated with the energy needed to manufacture the devices (this is embedded in the cost), disposal of small amounts of waste materials from the manufacturing process, and the land use for the installation of largescale PV systems. The impact of land use can be minimized by locating large-scale arrays in desert regions of little ecological importance and can be completely negated by integrating PV systems into existing building structures and rooftops.
A photovoltaic device, or solar cell, converts absorbed photons directly into electrical charges that are used to energize an external circuit (Figure 1). The photovoltaic effect was discovered in 1839 by French physicist Edmond Becquerel. Attempts at commercialization did not begin until a century later, and the first crystalline silicon PV device was developed in 1954 at Bell Laboratories.

A typical conventional solar cell is fabricated from an inorganic semiconductor material, such as crystalline $\mathrm{Si}$, that is doped to form a $p-n$ junction. The $p$ side contains an excess of positive charges (holes), and the $n$ side contains an excess of negative charges (electrons). In the region near the junction, called the depletion region, an electric field is formed. Electrons and holes generated through light absorption in the bulk of the Si diffuse to this junction, where they are accelerated by the electric field toward the proper electrode. If the $\mathrm{Si}$ is of sufficient quality, the charges reach the electrodes and leave the device in order to drive the external circuit. The power conversion efficiency of this process is defined as the ratio of the electric power provided to the external circuit to the solar power incident on the active area of the device. This is typically measured under standard simulated solar illumination conditions, and efficiencies of over $24 \%$ have been achieved in the laboratory for devices such as the one just described. As will be discussed later in this article, and throughout this issue of $M R S$ Bulletin, the fundamental mechanism by which organic PV devices work is substantially different than that of Si and other inorganic semiconductor devices.

In 2002, 49.3 quads ( 1 quad $=1$ quadrillion Btu $=2.9 \times 10^{11} \mathrm{kWh}$ ) of electrical energy were consumed worldwide. ${ }^{1}$ The U.S. accounted for the largest percentage of consumption (25.6\%), followed by Western Europe (19.2\%), China (10.2\%), Japan $(6.8 \%)$, and Russia (5.5\%).

As a case study, let's examine how much land area of PVs would be required to supply

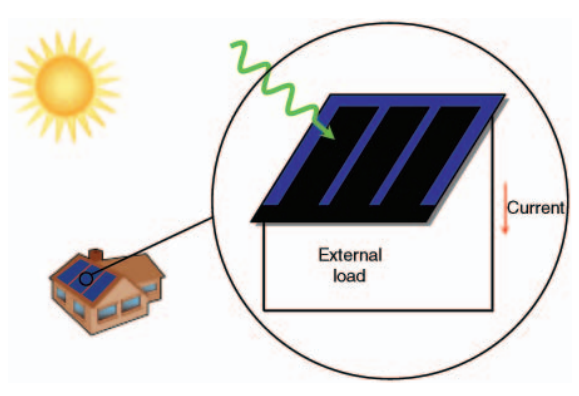

Figure 1. Illustration of the operation of a photovoltaic module. 
the electricity needs of the U.S., the largest consumer. In 2003, 39.6 quads of energy, largely from fossil fuels, were consumed to produce electricity in the U.S. After conversion losses, 13.1 quads of net electrical energy were output by power plants for general consumption. ${ }^{2}$ This amount of electricity could be produced by a $100 \mathrm{~km} \times 100 \mathrm{~km}$ land area in a region of high solar insolation, such as in the deserts of the Nevada or Arizona, covered with solar modules with a power conversion efficiency of $15 \%$. In order to meet the U.S. Department of Energy's cost goal of $\$ 0.33 / \mathrm{W},{ }^{3}$ these modules would have to be manufactured at a cost of $\$ 50 / \mathrm{m}^{2}$ or less. Similar calculations can be done for desert regions around the globe.

\section{Photovoltaic Technologies}

Given the vast potential of photovoltaic technology, worldwide production of terrestrial solar cell modules has been growing rapidly over the last several years, with Japan recently taking the lead in total production volume (Figure 2). Current production is dominated by single-crystal and polycrystalline silicon modules, which represent $94 \%$ of the market. These devices, based on silicon wafers, have been termed the "first generation" of PV technology. These are single-junction devices that are limited by thermodynamic considerations, stemming largely from energy loss due to carrier thermalization, to a maximum theoretical power conversion efficiency of $\sim 31 \%$ when illuminated by the AM 1.5 solar spectrum $^{+}$with an intensity of $1000 \mathrm{~W} / \mathrm{m}^{2}$ (1 sun). ${ }^{4}$ Progress in efficiencies of researchscale crystalline PV devices over that last several decades is shown in Figure 3. Clearly, significant improvements in device efficiencies have been steadily achieved.

One key to the development of any photovoltaic technology is the cost reduction associated with achieving economies of scale. This has been evident with the development of crystalline silicon PVs and will presumably be true for other technologies as their production volumes increase. Figure 4 shows the decrease in the cost of crystalline silicon PV modules as

\footnotetext{
${ }^{\dagger}$ Solar spectra are defined by an air mass (AM) value, which is a measure of the length of the path through the earth's atmosphere that the solar radiation travels. The value is calculated as $1 / \cos z$, where $z$ is the zenith angle between a line perpendicular to the earth's surface and a line intersecting the sun. AM 1 describes the case in which the sun is directly overhead. The AM 1.5 spectrum is commonly used for testing photovoltaic devices meant for terrestrial use. AM 0 is the spectrum of sunlight outside the earth's atmosphere and is used for testing PV devices intended for use in space.
}

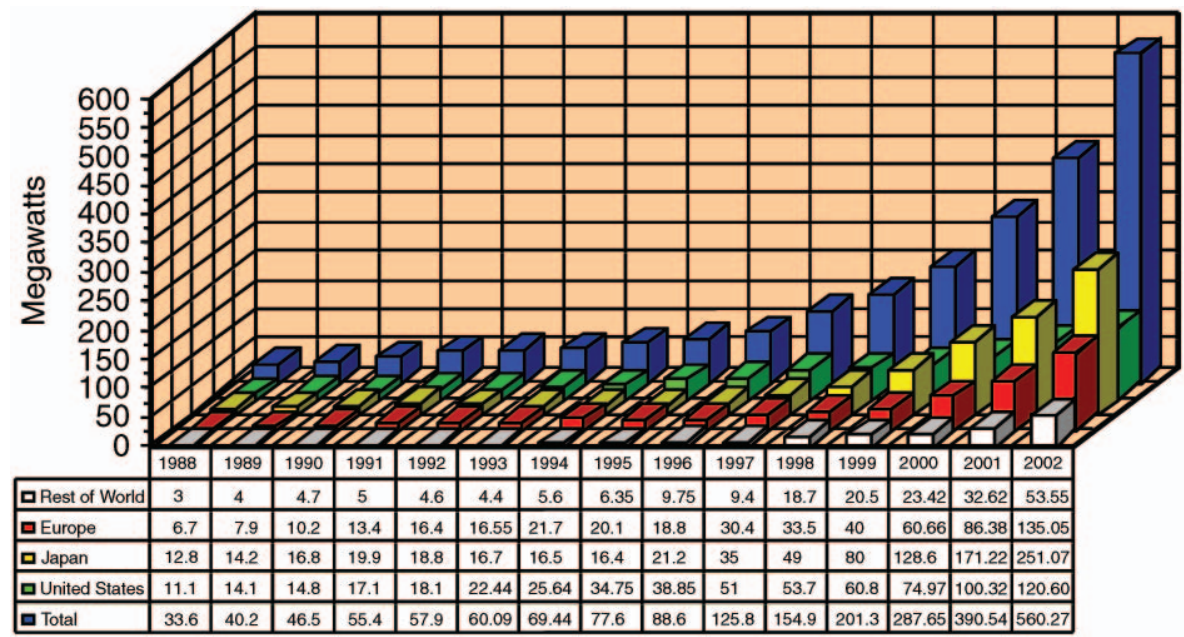

Figure 2. World photovoltaic module production (megawatts), total consumer and commercial use, per country. ${ }^{18}$ the production rate has increased, as well as predicted future costs for both c-Si on wafer and the emerging c-Si film on glass technologies. The current cost of $\sim \$ 4 / \mathrm{W}$ is still too high to significantly influence energy production markets. Best estimates are that costs will level off in the region of $\$ 1 / \mathrm{W}-\$ 1.50 / \mathrm{W}$ in the next 10 years, substantially higher than the $\$ 0.33 / \mathrm{W}$ target.

Thus, over the last decade, there has been considerable effort in advancing thinfilm, "second-generation" technologies that do not require the use of silicon wafer substrates and can therefore be manufactured at significantly reduced cost. ${ }^{5}$ Steady progress has been made in laboratory effi- ciencies, as can be seen in Figure 3 for devices based on $\mathrm{CdS} / \mathrm{CdTe}, \mathrm{Cu}(\mathrm{In}, \mathrm{Ga}) \mathrm{Se}_{2}$ (CIGS), and multijunction a-Si/a-SiGe. These devices are fabricated using techniques such as sputtering, physical vapor cal vapor deposition. Multijunction cells based on a-Si/a-SiGe have been the most date because of their ability to be fabricated at relatively low cost. Several companies are manufacturing a-Si/a-SiGe modules using roll-to-roll processing on flexible stainless steel and other substrates that allow high-speed production as well as easy integration into roofing materials. deposition, and plasma-enhanced chemisuccessful second-generation technology to

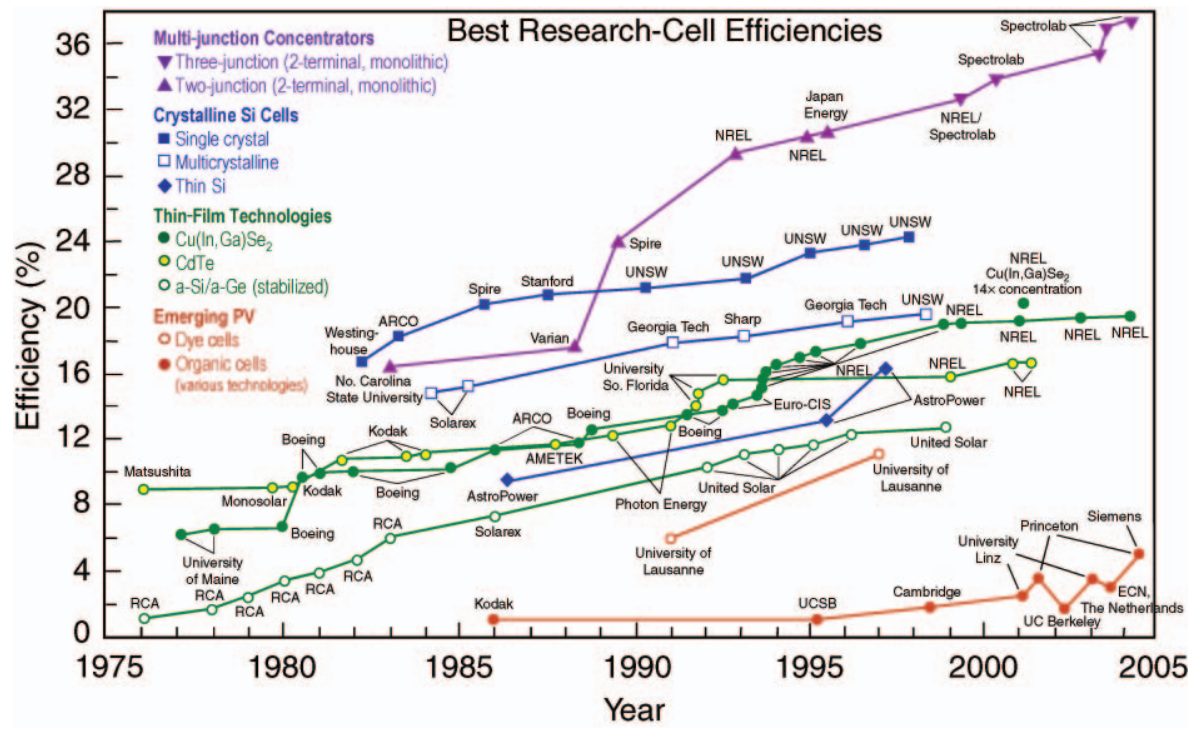

Figure 3. Progress of research-scale photovoltaic device efficiencies, under AM 1.5 simulated solar illumination, for a variety of technologies. 


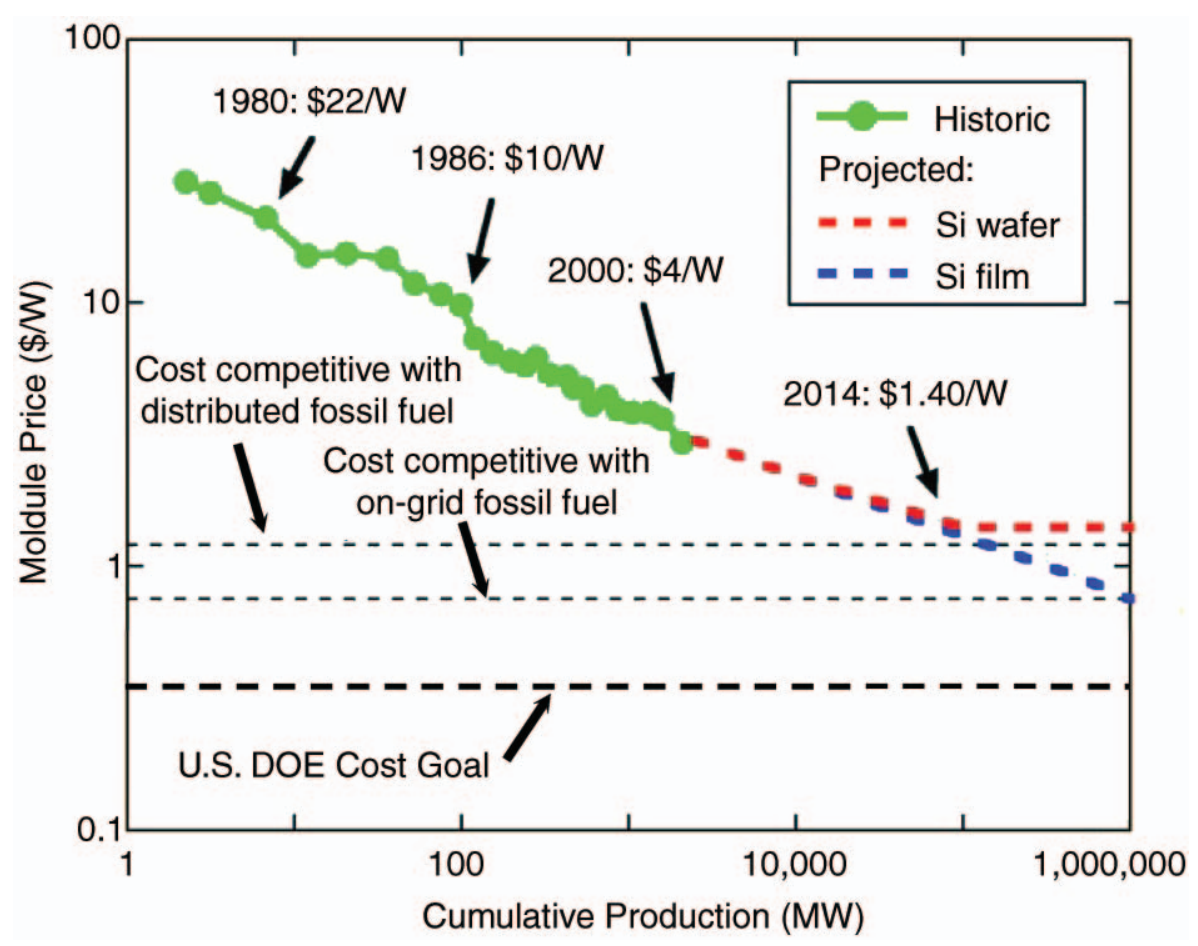

Figure 4. Historical and projected costs for wafer c-Si and film c-Si photovoltaic modules versus their cumulative production (in megawatts). "Distributed fossil fuel" refers to off-grid generation of power, using, for instance, gas-driven generators. ${ }^{19}$

These cells comprise $5.6 \%$ of the $6 \%$ of the market not dominated by crystalline or polycrystalline silicon.

The increasing manufacturing capacity in solar cells (as illustrated in Figure 4), coupled with the uncertainties of fossil fuel energy sources, is clearly a significant driver for the current PV technologies. Ultimately, however, there will be limitations on how much the costs of current technologies can be reduced. Achieving truly competitive cost/efficiency ratios may require breakthroughs even in mainstay technologies. This has engendered the concept of third-generation PV technologies, originally developed by Martin Green of the University of New South Wales and illustrated in Figure 5. ${ }^{6}$ Here, the thirdgeneration technologies (III) can be separated into two categories. In the first, IIIa, are novel approaches that strive to achieve very high efficiencies - by using concepts such as hot carriers, multiple electron-hole pair creation, and thermophotonics - that have theoretical maximum efficiencies well in excess of the $31 \%$ target for a singlejunction device. In this case, the allowed cost of the cell could be quite high. Research in this area is still in its infancy. Examples of the fundamental mechanisms have been demonstrated, but working devices have yet to be achieved. In the sec- ond type of third-generation device, IIIlb, the goal is to achieve moderate efficiency (15-20\%), but at a very low cost. To achieve this will require inexpensive materials for the active components and the packaging, low-temperature atmospheric processing, and high fabrication throughput. It is in this category that organic-based photo-

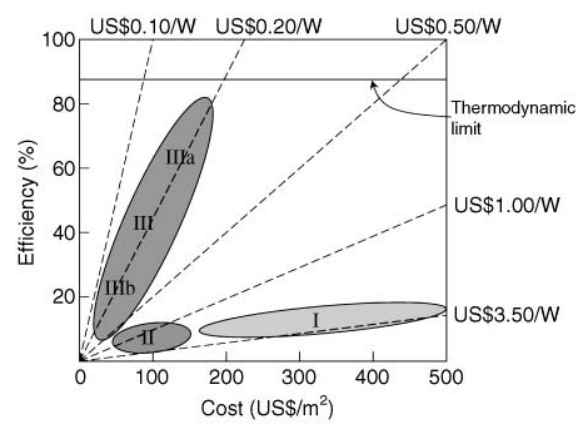

Figure 5. Cost-efficiency analysis for first-, second-, and third-generation photovoltaic technologies (labeled I, II, and III, respectively). Region IIIa depicts very-high-efficiency devices that require novel mechanisms of device operation. Region IIlb - the region in which organic $P V$ devices lie-depicts devices with moderate efficiencies and very low costs. ${ }^{20}$ voltaics (OPVs) have the potential to make a significant impact. Achieving these goals will require significant basic and applied science advancements over the next 10 years. This issue of MRS Bulletin will focus on the strengths and weaknesses of the different approaches that have been proposed for OPVs, along with the fundamental understanding required to fulfill their potential.

\section{Why Research the Development of Organic-Based Photovoltaics?}

With a theoretical efficiency that is the same as conventional semiconductor devices and a cost structure derived from plastic processing, organic photovoltaics offer the long-term potential of achieving the goal of a PV technology that is economically viable for large-scale power generation. The development of materials for OPVs can be leveraged by the increasing development of organics for transistors, actuators, packaging, waveguides, and displays. ${ }^{7,8}$ Some key advantages for OPVs are that organic small-molecule and polymer materials are inherently inexpensive; they can have very high optical absorption coefficients that permit the use of films with thicknesses of only several hundred nanometers; they are compatible with plastic substrates; and they can be fabricated using high-throughput, lowtemperature approaches that employ one of a variety of well-established printing techniques in a roll-to-roll process. ${ }^{9}$ Such low-temperature, and therefore low-energyconsuming, techniques require less capital investment than fabrication techniques for Si-based devices, for instance. In addition, the possibility of using flexible plastic substrates in an easily scalable, high-speed printing process can reduce the balanceof-system costs for OPVs to make them competitive with inorganic thin-film technologies. Thus, if efficiencies are comparable or even slightly lower than existing technologies, there may be compelling cost arguments favoring OPVs.

A key issue faced by the organic electronics community in general is the stability of the organic materials. It is encouraging that, for instance, automotive paints contain chromophores that are similar to molecules commonly used in OPV devices, and that organic light-emitting displays are demonstrating acceptable lifetimes under high injection currents. Device degradation pathways stem largely from changes in morphology, loss of interfacial adhesion, and interdiffusion of components, as opposed to strictly chemical decomposition. Thus, careful design and materials engineering can substantially improve device lifetimes. 
In addition to these factors, there are a number of other key aspects of organic materials. First and foremost is their versatility. They possess an unprecedented flexibility in the synthesis of the basic molecules, allowing for alteration of a wide range of properties, including molecular weight, bandgap, molecular orbital energy levels, wetting properties, structural properties (rigidity, conjugation length, moleculeto-molecule interactions, etc.), and doping. This ability to design and synthesize molecules and then integrate them into organic-organic and inorganic-organic composites provides a unique pathway in the design of materials for novel devices. Additionally, with the ability to alter the color of the device, fabricate devices on flexible substrates, and potentially print them in any pattern, OPVs may be integrated into existing building structures and into new commercial products in ways impossible for conventional technologies.

\section{The Nature of Organic Semiconductors}

Organic semiconductors (OSCs) can be broadly classified as either small molecules, with molecular weights of less than a few thousand atomic mass units, or polymers, with molecular weights greater than 10,000 amu. A more recent class of materials, called dendrimers, has also been developed that span a molecular weight range between small molecules and polymers.

The distinctions between these classes become most important in determining the processing that is required in making films and devices and for the subsequent molecular arrangements (morphologies) that are obtained. However, the fundamental mechanisms that underlie the optical and electronic properties of the different classes are identical. These processes are determined essentially by the molecular orbitals that are built up from the summation of individual atomic orbitals in the molecule. The frontier molecular orbitals, the highest occupied molecular orbital (HOMO) and the lowest unoccupied molecular orbital (LUMO), largely determine a molecule's properties. Figure 6 depicts the HOMOs of several common OSCs.

Photoexcitations in OSCs are inherently different than those in conventional inorganic semiconductors. ${ }^{10}$ Whereas light absorption in an inorganic semiconductor typically leads to the immediate production of free carriers (electrons and holes that are freely transported through the semiconductor material), light absorption in an OSC results in the formation of excitons (electron-hole pairs that are bound together by Coulomb attraction).

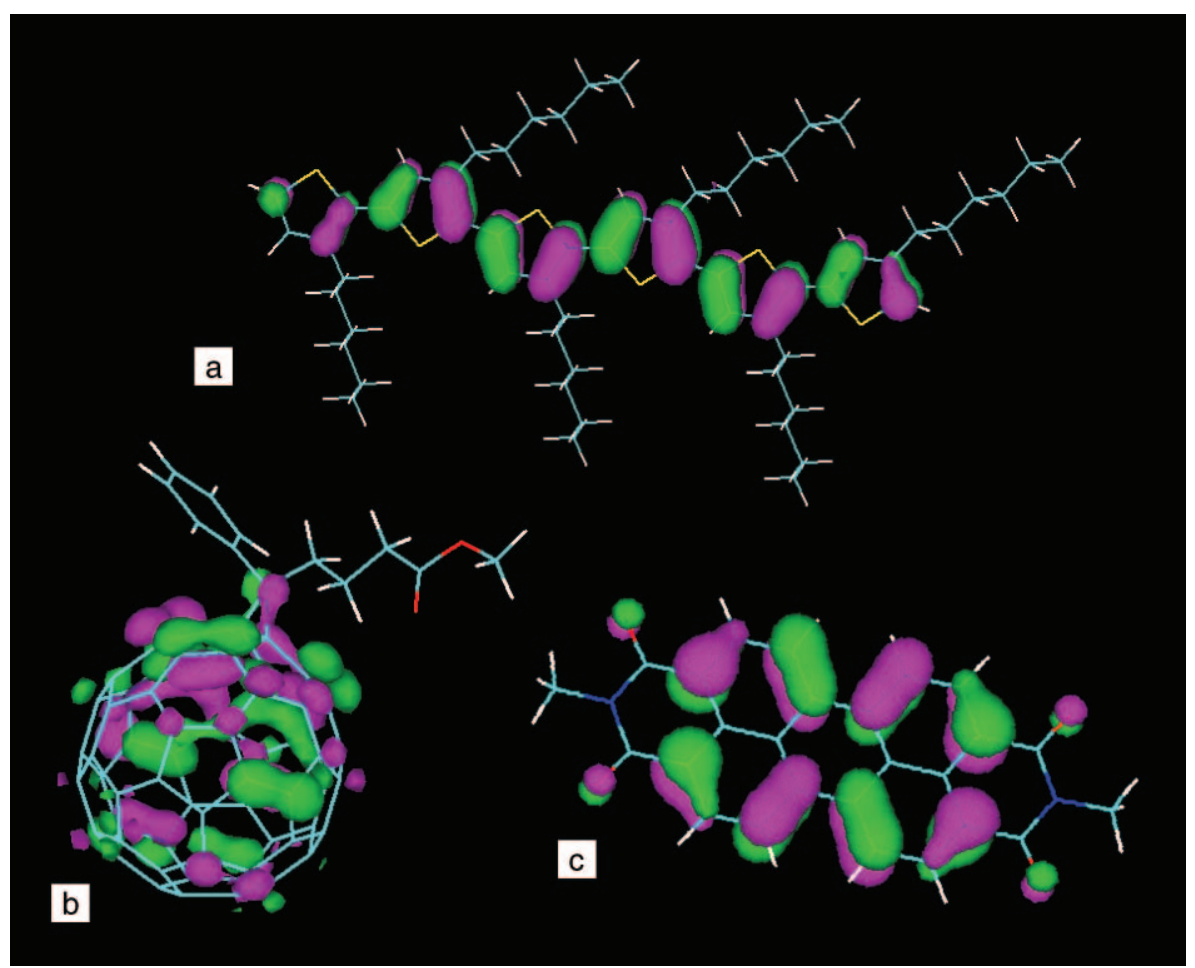

Figure 6. Depiction of the highest occupied molecular orbitals of several common organic semiconductors: (a) poly(3-hexylthiophene) (P3HT), (b) [6,6]-phenyl $C_{61}$-butyric acid methyl ester (PCBM), and (c) N,N'-dimethyl-3,4,9,10-perylene bis(tetracarboxyl diimide) (MPP). Green and pink regions depict positive and negative orbitals, respectively.

In order for free carriers to be generated in this case, the excitons must be dissociated. This can happen in the presence of high electric fields, at a defect site in the material, or at the interface between two materials that have a sufficient mismatch in their energetic levels (band offset). In the case of dissociation at the interface between two materials, an exciton created in either material can diffuse to the interface, leading to either electron transfer from the donor material to the acceptor material or hole transfer from the acceptor to the donor.

Thus, one can fabricate a photovoltaic device with the structure positive electrode/donor/acceptor/negative electrode. Tang, using a copper phthalocyanine layer as the donor and a perylene derivative as the acceptor, first accomplished this. ${ }^{11}$ This device had a power conversion efficiency of about 1\% under simulated solar illumination. As a result of the excitonic nature of the photoexcitations in OSCs, the operational mechanisms of OPVs are different from those of conventional photovoltaics. These differences are described in detail in the article in this issue by Gregg.

Charge transport in OSCs is determined by the intermolecular overlap of the fron- tier orbitals of adjacent molecules. ${ }^{12}$ This coupling is very weak compared with the interatomic coupling of conventional semiconductors. Additionally, the arrangement of molecules in a typical OSC is disordered and more resembles an amorphous mixture than a crystalline lattice. As a result, the conventional band transport description of charge transport cannot be used for OSCs. Instead, descriptions of charge transport in OSCs are based on carrier hopping between molecules. This is a temperatureactivated process that results in an increase in carrier mobility with increasing temperature, in contrast to the decreasing temperature dependence of the carrier mobility in conventional semiconductors.

It has also been found that carrier mobilities in OSCs follow an exponential dependence on the square root of the electric field. ${ }^{13}$ The temperature and field dependence for both small-molecule and polymer OSCs has been successfully modeled using what is known as the disorder formalism, originally developed by Bässler. ${ }^{14}$ This model assumes carrier hopping between molecular sites with Gaussian distributions of energies and geometric disorder parameters. A more recent version of the model has been developed that takes into account spatial correlations in the site energies and 
results in a better fit to experimental data over a wider range of electric fields. ${ }^{15}$ Typical carrier mobilities in OSCs range from $10^{-6} \mathrm{~cm}^{2} \mathrm{~V}^{-1} \mathrm{~s}^{-1}$ to $10^{-3} \mathrm{~cm}^{2} \mathrm{~V}^{-1} \mathrm{~s}^{-1}$. While these values are several orders of magnitude less than those found in conventional semiconductors, they are sufficiently high to allow for the efficient extraction of charges in OPVs before free carriers recombine to the ground state. Higher carrier mobilities have in fact been measured in crystalline OSCs-in materials such as anthracene or pentacene. These materials have been shown to be able to exhibit true band transport. However, there has not been a large focus on using such systems in OPV devices, since the slow growth techniques required to produce the crystalline lattice offset some of the cost benefits of organics and because additional problems arise with the presence of grain boundaries in these materials.

In trying to develop high-efficiency OPV devices, it has become clear to researchers in the field that the details of the morphology of the molecules have a large impact on the charge carrier mobilities and, therefore, device efficiencies. Learning how to control exactly where along the scale from amorphous to crystalline the structure of the material lies is important. Several techniques for doing this have been developed, including choice of solvent and casting conditions, thermal annealing, and interpenetration of the organic semiconductor into an inorganic lattice that influences the molecular alignment. ${ }^{15}$

\section{Paradigms for OPV Devices}

The molecular nature of organic semiconductors places constraints on the ways in which OPV devices must be designed. As a further consequence of the weak intermolecular coupling between molecules, the exciton diffusion length, which is the distance an exciton will travel on average before it decays to the ground state, is typically on the order of $10 \mathrm{~nm}$. This requires that planar devices, such as the Tang cell discussed earlier, consist of very thin active layers. Alternatively, the donor and acceptor species can be blended on the nanoscale such that all excitons are produced within approximately $10 \mathrm{~nm}$ of an interface. The low values for charge carrier mobilities in OSCs dictate that the thickness of the active layer be kept thin, thus limiting the optical density and the power conversion efficiency.

In this issue of MRS Bulletin, a variety of schemes to fabricate efficient OPV devices are discussed. These encompass different approaches to absorbing the sun's emission and accommodating the length scales derived from exciton diffusion lengths and carrier lifetimes in organic semiconductor materials. These approaches include - Dye-sensitized nanostructured oxide cells. These cells use an organic dye to absorb light and rapidly inject electrons into a nanostructured oxide such as anatase $\mathrm{TiO}_{2}$. The hole is scavenged by a redox couple, such as iodide/triiodide $\left(\mathrm{I}^{-} / \mathrm{I}_{3}^{-}\right)$, in solution or by a solid-state organic semiconductor or ionic medium. To date, the solid-state approaches have not been as effective, but progress is being made in understanding the nature of the redox behavior of the organic hole transporter. These approaches are discussed in the article by Grätzel.

- Multilayer devices in which smallmolecule OSCs are deposited sequentially to form a stacked device. Recent progress in the reduction of the series resistance has led to increased efficiencies. This technology has the added benefit that multijunction tandem devices, which trade off current for voltage and possess higher theoretical efficiencies, can be fabricated in a straightforward manner. These devices are discussed in the article by Forrest.

- Organic-organic composites in which donor and acceptor species, which function as the hole and electron transporters, respectively, are intimately mixed to produce a "bulk heterojunction." Among the most successful bulk heterojunction devices to date are those employing a conjugated polymer such as poly(3-hexylthiophene) as the donor and a fullerene derivative as the acceptor. These devices are described in the article by Janssen et al.

- Organic-inorganic composites that combine a light-absorbing conjugated polymer as the donor and hole transporter with a nanostructured, large-bandgap inorganic semiconductor such as $\mathrm{TiO}_{2}$ or $\mathrm{ZnO}$ as the acceptor and electron transporter. These work similarly to the bulk heterojunctions; however, the gross morphology of the mixture is determined by that of the nanostructured oxide that is grown in a selforganizing manner on the electrode. Optimization of the organic-inorganic interface is critical for these devices, which are discussed in the article by Coakley et al. - Organic-inorganic composites consisting of nanocrystals of conventional semiconductors, such as CdSe, blended into a conjugated polymer matrix. In this case, both components can efficiently absorb light, and the bandgap of the nanocrystals can be tuned by growing them to different sizes. As in the previous case, the energetics of the organic-inorganic interface is crucial. Recent progress in this area is discussed in the article by Milliron et al.

- Photoelectrochemical cells employing a conjugated polymer as the light absorber and electron donor in conjunction with a liquid electrolyte as the redox mediator and electron transporter. These devices, which are in very early stages of development, are discussed by Wallace et al.

\section{Industrial Interest}

Fueled by recent progress in the power conversion efficiency of OPV devices and the cost benefits of processing and fabricating organics, many companies have begun research on developing OPV devices to bring them to the marketplace. A partial list of companies performing research and development on OPV devices includes General Electric, Konarka, Nanosys, Nanosolar, Solaronix, Shell, Sharp, Sony, CDT, and Toshiba.

The article by Brabec et al. discusses the potential for high-throughput industrial manufacture of OPV modules. Solutionbased techniques such as inkjet, screen, or flexographic printing (a form of rotary web letterpress using flexible relief plates) are well suited for organic thin-film deposition and can be done under atmospheric conditions. The scalability and low cost of such techniques could alter the paradigm for solar cell manufacturing and could open new markets that make use of very inexpensive photovoltaics requiring only moderate efficiencies and device lifetimes.

An important economic factor in the development of any PV technology is the balance-of-system costs. As has been brought to light for thin-film technologies, the material costs of the active components will soon compose only a small part of the total module manufacturing costs. ${ }^{17}$ Glass substrates, transparent conducting electrodes (TCOs), and packaging materials instead will constitute a significant fraction of the final cost. In this area, reelto-reel manufacture of OPV modules can have an impact by using low-cost plastic substrates and conducting polymer alternatives to TCOs. Packaging is likely to be a major concern for the development of any practical organic semiconductor device, due to sensitivity to oxygen and water vapor. Progress has been made in this area with the development of flexible encapsulants for packaging organic light-emitting diodes. Industrial efforts into the development of OPVs can build on the success of such efforts to commercialize organic lightemitting diodes. Also encouraging is that initial efforts to fabricate OPV devices using high-throughput techniques show no decrease in performance over smallscale laboratory techniques.

\section{Future Challenges for Organic Photovoltaics}

Many formidable challenges must be overcome before OPV devices can be 
considered a truly practical technology. First and foremost, higher power conversion efficiencies must be demonstrated. Several groups are now reporting values in the $5 \%$ regime. These are very encouraging results; however, efficiencies of laboratory-scale devices must be pushed higher before largescale manufacturing can really be considered. Several fundamental issues must be addressed in order for this to happen. These include

- Bandgap. Current OPV devices have exhibited high $(>70 \%)$ quantum efficiency, defined as the ratio of the number of photons of a given wavelength incident on the device to the number of charges delivered to the external circuit, for blue photons. However, efficiently harvesting red photons, which contain a significant fraction of the energy in the solar spectrum, has proven challenging. Optical bandgaps of the lightabsorbing components of OPVs must be reduced to approach the nominally optimal value of $1.4 \mathrm{eV}$ while retaining good charge carrier mobilities.

- Interfaces. An improved understanding of the fundamental processes of charge transfer and carrier recombination across donoracceptor interfaces is critical. Here, the band offset must be optimized to yield the highest possible photovoltage from the device. Contact resistance between layers must be minimized in order to reduce the device series resistance, which is important in determining the fill factor and thus the power conversion efficiency. Additionally, physical properties such as wetting between organic and inorganic components and adhesion between layers are important for both the device performance and lifetime. - Charge transport. Obtaining higher charge carrier mobilities will allow for the use of thicker active layers while minimizing carrier recombination and keeping the series resistance of the device low. The development of organic molecules and organicinorganic composites that self-assemble into ordered phases with pathways for efficient charge transport is a promising route to obtaining higher carrier mobilities.

Devising new ways to address these issues, control the morphologies, and im- prove the optoelectronic properties of the light-absorbing and charge-transporting materials is the primary materials science thrust in all of the varieties of OPV devices. In addition to overcoming these fundamental obstacles to higher efficiency, pathways of device degradation must be identified and mitigated before device lifetimes can be sufficiently long for practical use. Manufacturing issues such as printing and/or lamination of electrode materials, as opposed to vacuum deposition of metal electrodes, must be researched, and optimization of device patterning and integration of OPV devices into module assemblies must also be done.

As a final note, we mention that most of the efficiency values reported or claimed for OPV devices have not yet been certified by an internationally recognized PV test and measurement laboratory, such as the National Renewable Energy Laboratory in Golden, Colo. Photovoltaic device efficiencies are notoriously difficult to measure accurately because, among other reasons, of the sensitivity of the device performance to deviations between the emission spectrum of the solar simulator used in testing and the true AM 1.5 spectrum. Until recently, certified measurements of OPV efficiencies have not been critical, since efficiency values in the field have been relatively low. Recent developments to push efficiencies into the $5 \%$ regime, which is approaching that of amorphous-siliconbased devices, now make accurate measurements more important. These advances are a testament to the rapid progress being made in the field of organic photovoltaics and to the promise they hold for growing into a viable technology as a low-cost renewable energy resource.

\section{Acknowledgments}

The authors thank Dr. Howard Branz, Dr. Brian Gregg, and Dr. Garry Rumbles at NREL for fruitful discussions and continued collaborations. S.E. Shaheen and D.S. Ginley gratefully acknowledge the financial support for research in organic photovoltaics through the NREL DDRD program, DARPA, and the Xcel Energy Renewable
Development Fund. G.E. Jabbour would like to thank NREL for financial support.

\section{References}

1. International Electricity Information, U.S. Dept. of Energy, http://www.eia.doe.gov/emeu/ international/electric.html\#Consumption (accessed December 2004).

2. Annual Energy Review 2003, U.S. Dept. of Energy, http://www.eia.doe.gov/emeu/aer/ contents.html (accessed December 2004).

3. U.S. Photovoltaic Industry Roadmap, U.S. Dept. of Energy, http://www.nrel.gov/ncpv/ pvmenu.cgi site $=n c p v \& i d x=3 \& b o d y=$ infores.html (accessed December 2004).

4. W. Shockley and H.J. Queisser, J. App. Phys. 32 (3) (1961) p. 510.

5. K. Zweibel, B. Von Roedern, and H. Ullal, "Finally: Thin-film PV!" Photon Int. (October 2004) p. 48.

6. M.A. Green, Physica E 14 (1-2) (2002) p. 65 .

7. G. Collins, Sci. Am. (August 2004) p. 74.

8. D.M. de Leeuw, Phys. World 12 (3) (1999) p. 31.

9. S.E. Shaheen, R. Radspinner, N. Peyghambarian, and G.E. Jabbour, Appl. Phys. Lett. 79 (18) (2001) p. 2996.

10. B.A. Gregg and M.C. Hanna, J. Appl. Phys. 93 (6) (2003) p. 3605.

11. C.W. Tang, Appl. Phys. Lett. 48 (2) (1986) p. 183. 12. J.L. Bredas, J.P. Calbert, D.A. da Silva Filho, and J. Cornil, Proc. Nat. Acad. Sci. 99 (9) (2002) p. 5804.

13. P.W.M. Blom, M.J.M. de Jong, and M.G. van Munster, Phys. Rev. B 55 (2) (1997) p. R656.

14. H. Bässler, Phys. Status Solidi B 175 (1993) p. 15.

15. S.V. Novikov, D.H. Dunlap, V.M. Kenkre, P.E. Parris, and A.V. Vannikov, Phys. Rev. Lett. 81 (20) (1998) p. 4472.

16. S.E. Shaheen and D.S. Ginley, "Photovoltaics for the Next Generation: Organic-Based Solar Cells," in Encyclopedia of Nanoscience and Nanotechnology, edited by J.A. Schwarz, C. Contescu, and K. Putyera (Marcel Dekker, New York, 2004), p. 2879.

17. K. Zweibel, Sol. Energy Mater. Sol. Cells 63 (4) (2000) p. 375.

18. P. Maycock, PV News (February 2003).

19. R.M. Margolis, "Photovoltaic Technology Experience Curves and Markets," NCPV Solar Program Review Meeting, Denver, CO (2003).

20. M.A. Green, "Third generation photovoltaics: concepts for high efficiency at low cost," Proc. Electrochem. Soc. 10 (2001) p. 3.

\begin{abstract}
Sean E. Shaheen, Guest Editor for this issue of MRS Bulletin, has been a researcher for the National Center for Photovoltaics at the National Renewable Energy Laboratory since 2002, where he works on the development of novel materials

for organic-based solar cells and simulation and modeling of the physics of these devices. He received his $\mathrm{PhD}$ in physics from the University of Arizona (1999) and performed postdoctoral research at the University of Linz, Austria,
\end{abstract}

as a Lise Meitner Postdoctoral Fellow. His research interests extend to modeling of information processing in biological and other complex dynamical systems.

Shaheen can be reached by e-mail at sean_shaheen@nrel.gov.
David S. Ginley, Guest Editor for this issue of MRS Bulletin, is a Group Manager in Process Technology and Advanced Concepts at the National Renewable Energy Laboratory, leading activities in the applications of nanotechnology, organic electronics, transition-metal oxides (ferroelectric materials, rechargeable Li batteries, and transparent conductors) and inkjet printing. He is also an adjunct professor at the University of Colorado at Boulder (physics) and the Colorado School of Mines (materials sci- 


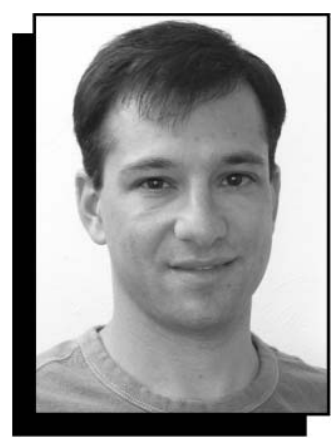

Sean E. Shaheen

ence). His work focuses on the development and basic science of transparent conducting oxides, ferroelectric materials, organic materials, and nanomaterials and the development of nextgeneration process technology for materials and device development (combinatorial methods, direct-write materials, composite materials, and non-vacuum processing).

Ginley holds a BS degree in mineral engineering chemistry from the Colorado School of Mines and a $\mathrm{PhD}$ degree in inorganic chemistry from MIT. Ginley is a fellow of the Electrochemical Society. He has published more than 320 papers and holds 24 patents. He has been honored with a Department of Energy Award for Sustained Research in Superconducting Materials and R\&D 100

Awards for novel chemical etches, nanoparticle technology, ferroelectric frequency agile electronics, and alumina-based nanofibers. He has also received two FLC technology transfer awards.

Ginley can be

reached by e-mail at david_ginley@nrel.gov.

Ghassan E. Jabbour, Guest Editor for this issue of MRS Bulletin, is a professor of chemical and materials engineering and the technical area leader of optoelec-

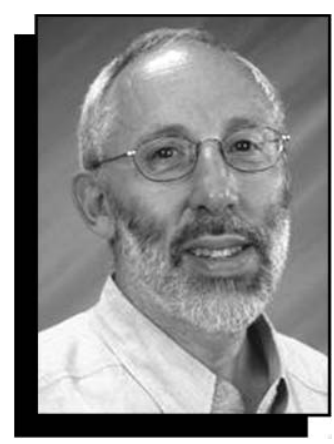

David S. Ginley

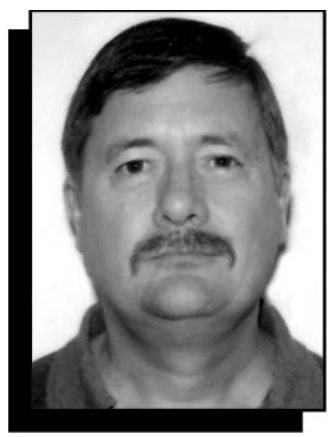

Brian A. Gregg

tronic materials and devices at the Flexible Display Center (FDC) at Arizona State University. Jabbour is also leads the Technical Advisory Board on Optoelectronic Materials, Devices, and Encapsulation at FDC.

His research focuses on the fabrication, characterization, and testing of organic (polymeric and small-molecule) electronics and photonics. His group was the first to demonstrate the use of high-throughput printing techniques in the fabrication of organic solar cells and diodes.

Jabbour holds a PhD degree in materials science and engineering from the University of Arizona. He is associate editor of the Journal of the Society for Information Display (JSID). He was the track chair of the

Nanotechnology Program for the SPIE Annual Meeting (2001-2004), and the secretary general for the Materials Secretariat

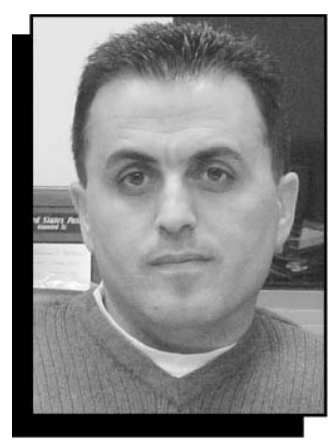

Ghassan E. Jabbour

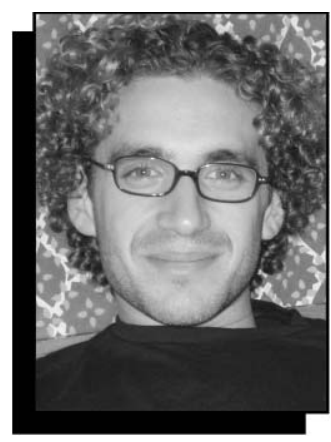

Ilan Gur

of the American Chemical Society (2001). Jabbour has been involved as a chair, co-chair, or committee member for more than 40 conferences related to photonic and electronic properties of organic materials and their applications in displays and lighting, transistors and solar cells, hybrid photosensitive materials, and hybrid integration of semiconducting materials. He is an SPIE fellow and was named one of the $100 \mathrm{New}$ Leaders of the USA in 2001 by Asahi Shimbun (Japan).

Jabbour can be reached by e-mail at jabbour@asu.edu and by telephone at 480-727-8930.

\section{A. Paul Alivisatos is a} professor of materials science at the University of California, Berkeley, and director of the Materials Sciences Division. $\mathrm{He}$ is also the scientific director of the Molecu-

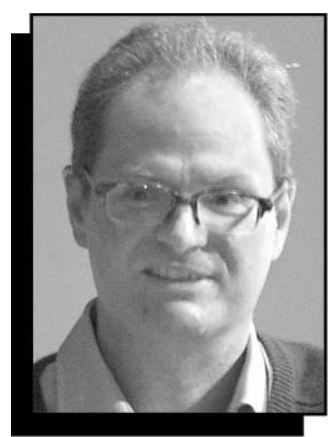

A. Paul Alivisatos

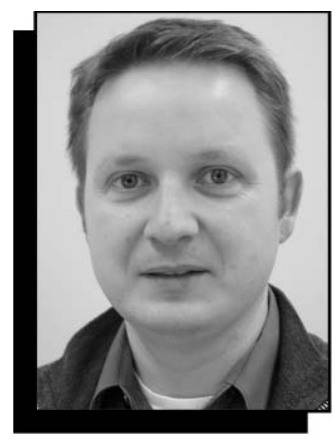

Jens A. Hauch

lar Foundry Program at the Lawrence Berkeley National Laboratory (LBNL) and holds a senior scientist appointment at LBNL. In the private sector, Alivisatos is the founder and editor in chief of Nano Letters, and the scientific founder of Quantum Dot Corp. and Nanosys Inc.

Alivisatos' research seeks to understand the synthesis and characterization of semiconductor nanocrystals. He holds a BA degree in chemistry from the University of Chicago and $\mathrm{PhD}$ degree in chemical physics from UC-Berkeley. He held a postdoctoral appointment with Louis Brus at AT\&T Bell Laboratories and returned to Berkeley in 1988, where he joined the faculty as an assistant professor of chemistry. He was named associate professor in 1993, and became a full professor of chemistry in 1995.

He was also given the honor of being named

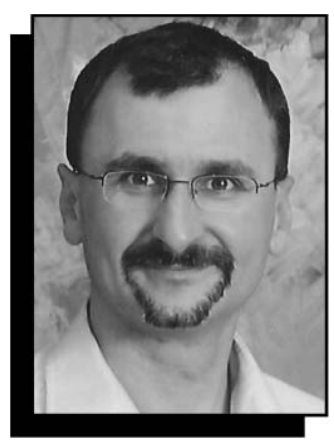

Christoph J. Brabec

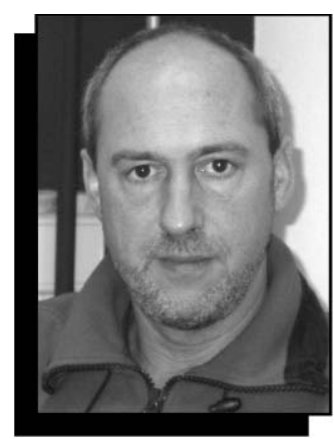

Jan C. (Kees) Hummelen

Chancellor's Professor of Chemistry from 1998 to 2001. In 1999, he was named professor of materials science.

He has received many awards for his contributions to the field of nanotechnology, the most recent being election into the National Academy of Sciences and the American Academy of Arts, and receiving the 2005 ACS Award in Colloid and Surface Chemistry.

Alivisatos can be reached by e-mail at alivis@uclink4.berkeley. edu.

Christoph J. Brabec is director of the polymer photovoltaics program at Konarka Technologies. Before he and his team joined Konarka, he was project leader at Siemens Corporate Technology, where he focused on the development of polymer electronic devices (solar cells, displays, imaging). While working on his PhD degree in 1995, he 


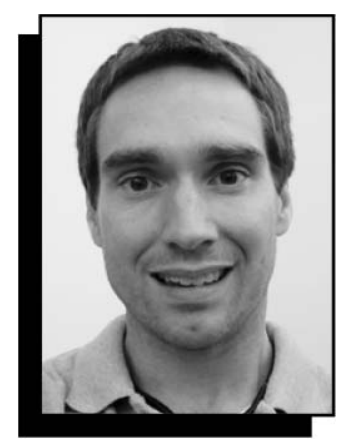

Kevin M. Coakley

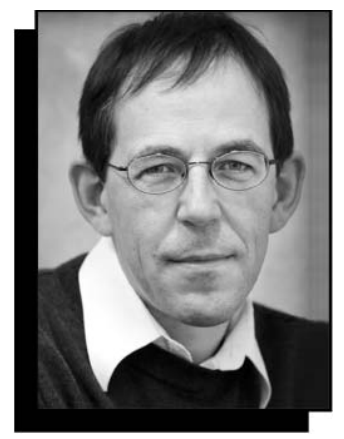

René A.J. Janssen

investigated the rheology of polymer melts with respect to molar mass correlations. In 1996, he joined the group of Alan Heeger at the University of Santa Barbara for a sabbatical, and he continued to work on the optoelectronic properties of organic semiconductors later on as an assistant professor at the University of Linz. In 1998, he worked as a senior scientist at the Christian Doppler Laboratory on organic solar cells, joining Siemens in 2001. He is author or coauthor of more than 100 papers and 30 patents; he finished his habilitation in physical chemistry in 2003.

Brabec can be reached by e-mail at cbrabec@ konarka.com.

Kevin M. Coakley is a recent $\mathrm{PhD}$ graduate from the Department of Materials Science and Engineering at Stanford University. His research there focused on fabri-

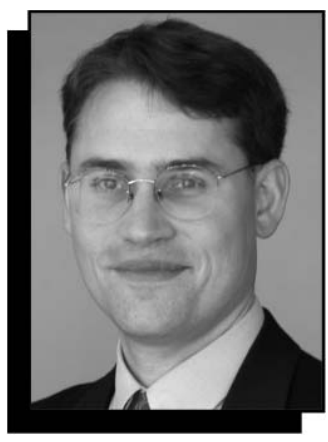

Paul C. Dastoor

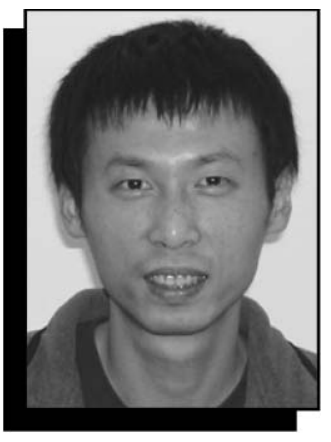

Yuxiang Liu

cating photovoltaic cells from conjugated polymers infiltrated into ordered nanoporous hosts and on the electrical and optical properties of conjugated polymers in confined channels.

Coakley can be reached by e-mail at kcoakley@stanford.edu.

Paul C. Dastoor is a senior lecturer in physics in the School of Mathematical and Physical Sciences at the University of Newcastle in Australia. He received his BA degree in natural sciences from the University of Cambridge in 1990 and his $\mathrm{PhD}$ degree in surface physics, also from the University of Cambridge, in 1995. After completing his doctorate, he joined the Surface Chemistry Department at British Steel in 1994 before taking up his present appointment at the University of Newcastle in 1995.

He was an EPSRC Visiting Research Fellow

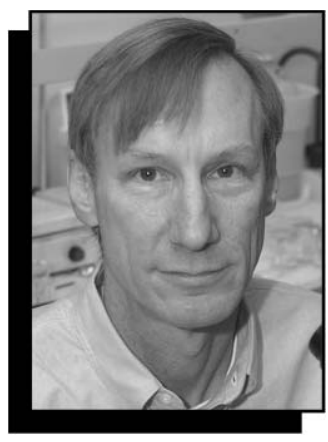

Stephen R. Forrest

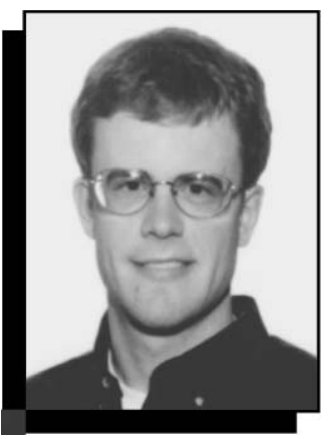

Michael D. McGehee

at Fitzwilliam College, Cambridge, in 2002 and a CCLRC Visiting Research Fellow at the Daresbury Laboratory, Cheshire, for 2004-2005. His research interests encompass the growth and properties of thin films, surface coatings, and organic electronic devices based on semiconducting polymers.

Dastoor can be reached by e-mail at phpd@alinga.newcastle. edu.au.

Stephen R. Forrest is the James S. McDonnell Distinguished University Professor of Electrical Engineering at Princeton University. He holds a BA degree in physics from the University of California and MSc and $\mathrm{PhD}$ degrees in physics from the University of Michigan. From there, he investigated photodetectors for optical communications at Bell Laboratories. In 1985, Forrest joined the Electrical Engineering and

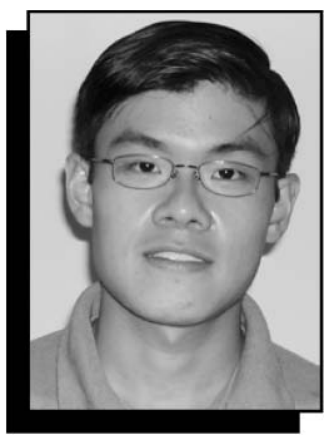

Chiatzun Goh

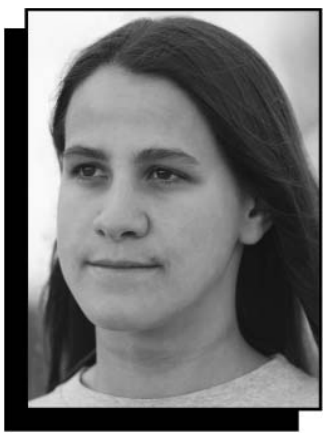

Delia J. Milliron

Materials Science Departments at the University of Southern California, where he worked on optoelectronic integrated circuits and organic semiconductors. He has served as director of the National Center for Integrated Photonic Technology as well as director of Princeton's Center for Photonics and Optoelectronic Materials (POEM). From 1997 to 2001, he was chair of the Electrical Engineering Department at Princeton. A fellow of the IEEE and OSA, and a member of the National Academy of Engineering, he received the IEEE/LEOS Distinguished Lecturer Award for 1996-1997, and in 1998, he was co-recipient of the IPO National Distinguished Inventor Award as well as the Thomas Alva Edison Award for innovations in organic LEDs. In 1999, Forrest received the MRS Medal for

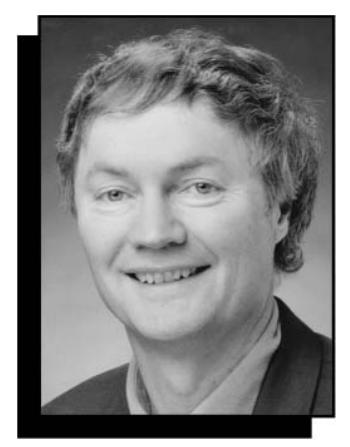

Michael Grätzel

work on organic thin films. In 2001, he was awarded the IEEE/LEOS William Streifer Scientific Achievement Award for advances made on photodetectors for optical communications systems. Forrest has authored approximately 365 papers in refereed journals and holds 122 patents.

Forrest can be reached by e-mail at forrest@ princeton.edu.

Chiatzun Goh is a $\mathrm{PhD}$ candidate in the Department of Materials Science and Engineering at Stanford University. His current research interests include the investigation of charge transport in conjugated polymers and nanopatterning of metal oxide semiconductors for use in organic photovoltaic cells. He holds a BA degree in chemistry from Beloit College and is currently a Kodak fellow.

Goh can be reached by e-mail at ctgoh@ stanford.edu.

Michael Grätzel is a professor at the Swiss Federal Institute of Technology in Lausanne, where he directs the Laboratory of Photonics and Interfaces, and he is a part-time distinguished visiting professor at the Delft University of Technology. He has pioneered research on nanomaterials 
and display-related applications, and he developed a new type of solar cell based on dyesensitized mesoscopic oxide particles.

Grätzel is the author of more than 500 publications, two books, and over 40 patents. He has been an invited professor at the University of California at Berkeley, the University of Tokyo, and the Ecole National Supérieur de Paris. He has received numerous awards, including the Millennium 2000 European innovation price, the 2001 Faraday Medal from the British Royal Society, the 2001 Dutch Havinga Award, the 2003 Italgas Prize, and McKinsey Venture Awards in 1998 and 2002. He holds a doctor degree from the TU Berlin and honorary doctorates from the Universities of Uppsala and Turin.

Grätzel can be reached by e-mail at michael.graetzel@epfl.ch.

Brian A. Gregg is a senior scientist at the National Renewable Energy Laboratory. He pioneered the use of liquid-crystalline organic semiconductors in photovoltaic cells, invented the self-powered "photoelectrochromic" smart window, and has made a number of contributions to understanding the kinetics, thermodynamics, and recombination processes in dye-sensitized solar cells and in solidstate organic PV cells. He has published more than 60 papers on organic photovoltaic cells in the last 17 years.

Gregg can be reached by e-mail at brian_gregg@nrel.gov.

Ilan Gur is a National Science Foundation graduate research fellow and $\mathrm{PhD}$ candidate in materials science and engineering at the University of California at Berkeley, where he has also earned BA and MS degrees. His scientific research interests are in the design of novel materials systems and device architectures to enable low-cost photovoltaic energy conversion, with a focus on II-VI semiconductor nanostructures and conjugated organic materials. He was the recipient of the National Defense Science and Engineering Graduate fellowship from the U.S. Department of Defense and the United Nations Industrial Development Organization's international research fellowship.

Gur can be reached by e-mail at igur@ berkeley.edu.

Jens A. Hauch is head of polymer photovoltaic production development at Konarka Technologies. He holds a BS degree in physics from the University of Illinois at Urbana-Champaign, where he was a member of the Center for Complex Systems Research. He holds a $\mathrm{PhD}$ degree from the Center for Nonlinear Dynamics at the University of Texas at Austin. Before joining Konarka in 2004, he was active in the development of thin-film magnetic sensors, electrochromic displays, and organic photodetectors at Siemens Corporate Technology.

Hauch can be reached by e-mail at jhauch@konarka.com.

Jan C. (Kees) Hummelen holds a master's degree in chemistry and a doctorate degree in science

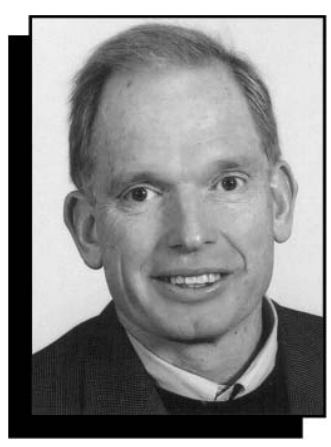

David L. Officer

from the University of Groningen, the Netherlands. After two years as a postdoctoral fellow in the Institute for Polymers and Organic Solids at the University of California, Santa Barbara, and six months at Eindhoven Technical University, he returned to Groningen, where he was appointed Universitair Hoofdocent in 1998 and a full professor of chemistry in 2000. Over the last 10 years, his main research activities have been in fundamental and applied fullerene chemistry, the development of plastic photovoltaic technology, and molecular and nanoelectronics.

Hummelen can be reached by e-mail at j.c.hummelen@chem. rug.nl.

René A.J. Janssen is professor of chemistry and physics at Eindhoven University of Technology. His group's research focuses on functional conjugated molecules, macromolecules, nanostructures, and materials aiming at electro-optical applications, using synthetic organic and polymer chemistry combined with advanced optical spectroscopy, electrochemistry, morphological characterization, and the preparation and analysis of prototype devices.

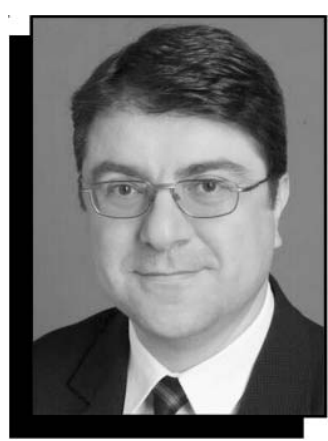

Niyazi Serdar Sariciftci

Janssen can be reached by e-mail at r.a.j.janssen@tue.nl.

Yuxiang Liu is a $\mathrm{PhD}$ candidate in the Department of Chemistry at Stanford University. His current research is focused on the synthesis of mesoporous metal oxides for photovoltaic applications and the study of exciton diffusion in organic semiconductors. He holds a BA degree in chemistry from Beijing University. Liu can be reached by e-mail at yxliu@ stanford.edu.

Michael D. McGehee is an assistant professor in the Materials Science and Engineering Department at Stanford University. He holds an $\mathrm{AB}$ degree in physics from Princeton University, and a PhD degree in materials science from the University of California at Santa Barbara, where he did research on polymer lasers in the laboratory of Alan Heeger. After graduation, he did postdoctoral research at UCSB and joined the Stanford faculty in 2000. His research interests have included organicinorganic nanostructures for applications such as photovoltaics, light extraction from polymer LEDs and the chemistry and physics

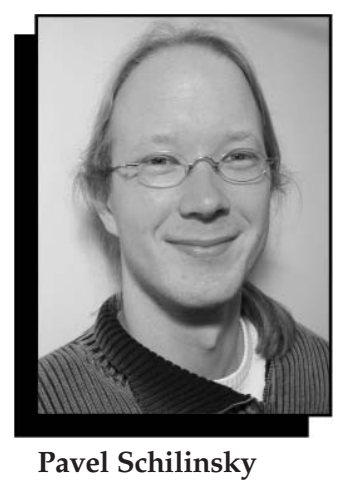

of organic semiconductors. McGehee has won a National Science Foundation CAREER Award, the Henry and Camille Dreyfus New Faculty Award and the Dupont Young Professor Award.

McGehee can be reached by e-mail at mmcgehee@stanford.edu.

Delia J. Milliron is pursuing postdoctoral studies at the IBM T.J. Watson Research Center. She holds an $\mathrm{AB}$ degree in chemistry with a certificate in materials science from Princeton University. She completed her $\mathrm{PhD}$ degree in chemistry at the University of California at Berkeley under A. Paul Alivisatos. Her research has involved next-generation electronic materials such as organic molecular crystals, semiconducting polymers, and inorganic nanocrystals. Her work has thus far included development of new materials, their application in light-emitting diodes, photovoltaic cells, and field-effect transistors, and advancing the understanding of dissimilar material interactions that take place in these devices.

Milliron can be reached by e-mail at milliron@us.ibm.com.

David L. Officer is director of the Nanomaterials 


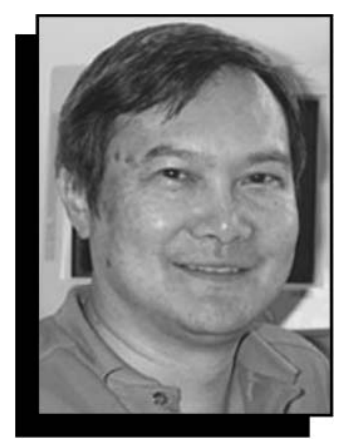

Chee On Too

Research Centre (NRC) and professor of chemistry at the Institute of Fundamental Sciences, Massey University. He is a principal investigator at the MacDiarmid Institute for Advanced Materials and Nanotechnology, a New Zealand Centre of Research Excellence, and he is a partner investigator with the Australian Research Council Centre for Nanostructured Electromaterials. He has collaborative research programs in the synthesis and use of porphyrins for photovoltaic and molecular devices and in the development and application of functionalized polythiophenes and carbon nanotubes. He holds a PhD degree from Victoria University of Wellington in New Zealand.

Officer can be reached by e-mail at d.officer@ massey.ac.nz.

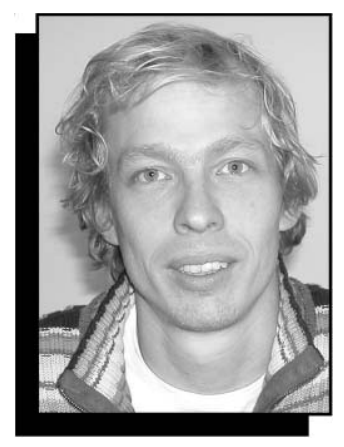

Christoph Waldauf

Niyazi Serdar Sariciftci is chair of the Institute for Physical Chemistry at Johannes Kepler University in Linz, Austria. $\mathrm{He}$ is also the founding director of the Linzer Institut für Organische Solarzellen (LIOS). He holds a BA degree from Sankt Georgs Kolleg in Istanbul, Turkey, and $\mathrm{MA}$ and PhD degrees in physics from the University of Vienna. He did his postdoctoral research at the University of Stuttgart's Physics Institute. From 1992 to 1996, he was a senior research associate at the Institute for Polymers and Organic Solids at the University of California, Santa Barbara, working in the areas of photoinduced optical, magnetic-resonance, and transport phenomena in conducting polymers.

Sariciftci can be reached by e-mail at serdar.sariciftci@ jk.uni-linz.ac.at.

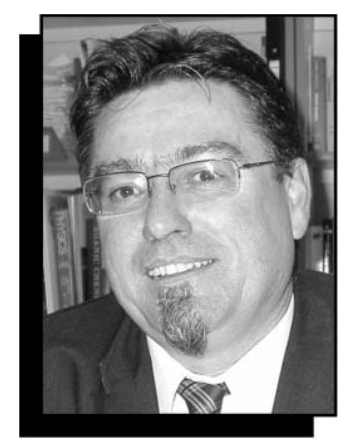

Gordon G. Wallace

Pavel Schilinsky is finalizing a PhD degree in physics from the University of Oldenburg. In August 2004, he began working at Konarka Technologies in Germany. He also studied physics at the University of Bremen and the Carl von Ossietzky University of Oldenburg and wrote his master's thesis on the topic of synthesis and characterization of $\mathrm{CdS}$ and CuS nanoparticles for photovoltaic applications.

Schilinsky can be reached by e-mail at pschilinsky@

konarka.com.

Chee On Too is the assistant director of the Intelligent Polymer Research Institute and chief operating officer of the Australian Research Council Centre for Nanostructured Electromaterials at the University of Wollongong, Australia. He has published 54 refereed pa- pers on the synthesis and processing of conducting polymers as well as on the applications of conducting polymers, including smart separation technologies (chromatography and membranes), sensors, controlledrelease and thermoresponsive conducting polymer composites, metal-ion separation using conductive electroactive polymer colloids, catalytic hydrogen generation, polymer batteries, and photovoltaics.

Too can be reached by e-mail at chee@ uow.edu.au.

Christoph Waldauf is a research and development scientist at Konarka Technologies, where he works on the development of organic photovoltaic efficiencies, lifetimes, and process optimization. He received his education from the Technical School for Electronics and Telecommunication, the Technical University of Graz, and the University of Oldenburg. He has been a site acquisition manager for Connect Austria, a technician at the department of Advanced Technology at Datacon, and a scientist at Siemens Corporate Technologies.

Waldauf can be reached by e-mail at cwaldauf@konarka.com.
Gordon G. Wallace is research director of the Australian Research Council Centre for Nanostructured Electromaterials. In 1990, he was appointed professor at the University of Wollongong. He holds a DSc degree from Deakin University. He was awarded an Australian Research Council (ARC) QEII Fellowship in 1991 and an ARC Senior Research Fellowship in 1995. He was appointed to an ARC Professorial Fellowship in 2002 and received an ETS Walton Fellowship from the Science Foundation Ireland in 2003. In the same year, he was elected fellow of the Australian Academy of Technological Sciences and Engineering. He is also a fellow of the Royal Australian Chemical Institute (RACI), and he received the inaugural Polymer Science and Technology Award from the RACI in 1992 and the RACI Stokes Medal for research in electrochemistry in 2004. Wallace has published more than 300 refereed publications and a monograph on inherently conducting polymers for intelligent materials systems.

Wallace can be reached by e-mail at gordon_wallace@ uow.edu.au.

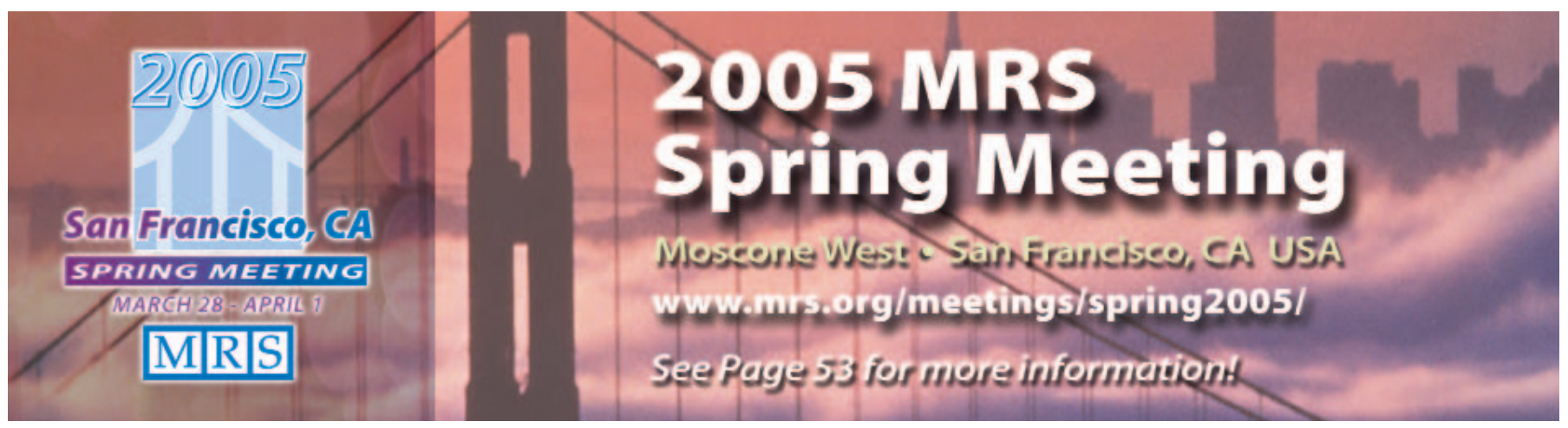

